\title{
Single Chip Wireless Condition Monitoring of Power Semiconductor Modules
}

\author{
Joakim Nilsson, Johan Borg, Jonny Johansson \\ Dept. of Computer Science, Electrical and Space Engineering, \\ Luleå University of Technology, Luleå, Sweden \\ Email: joanil@ltu.se
}

\begin{abstract}
A concept for doing accurate monitoring of temperature in power semiconductor modules is proposed. The concept involves glueing wireless single-chip temperature sensors with on-chip coils in direct contact with power semiconductor devices within their modules. Direct contact results in accurate temperature measurements while wireless technology such as RFID provides galvanic isolation from the power devices.

An overview of the electromagnetic situation within wire bond power semiconductor modules is presented and a prototype chip with an on-chip coil has been manufactured as an initial attempt to investigate the feasibility of the concept. Measurements on said chip provides some insight in the challenges in on-chip coil designs.

The feasibility of the concept is supported by earlier work that have demonstrated high power transfer efficiencies and a low power temperature sensor that is able to operate at high temperatures.
\end{abstract}

Index Terms-low power, wireless, RFID, on-chip coil, condition monitoring, power semiconductor

\section{INTRODUCTION}

A study conducted in 2007 shows that $34 \%$ of failures occurring in power electronic equipment are due to solder faults or faults in semiconductors [1]. Condition monitoring of power semiconductors can be used to predict such failures so that scheduled maintenance can be performed and catastrophic or secondary failures can be avoided. A rise in temperature can be an indication of an emerging fault and two studies suggest that monitoring of the power semiconductor temperature is a promising method for predicting emerging failures [2], [3].

This paper presents the idea of using wireless direct contact temperature sensors for use in condition monitoring of power semiconductors. Such sensors could make emerging faults in power semiconductors easier to predict by providing accurate measurements of temperature. This is especially true for faults related to the packaging of a device. A common package fault is solder fatigue, where the solder that attaches the power semiconductor to its cooling base plate degenerates, reducing the cooling efficiency which eventually results in the device becoming permanently damaged due to overheating [4].

A wireless approach solves the problem of providing galvanic isolation from the power semiconductor. Another approach is to take indirect measurements to provide galvanic isolation. Three indirect methods were demonstrated in [5] that estimate the junction temperature of a power MOSFET device by taking measurements of its various voltages and currents and calculating the estimated temperature from them.

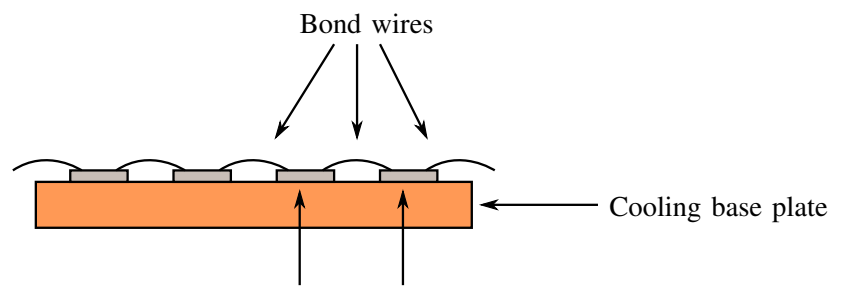

Power semiconductors

Fig. 1. Schematic view of the cross-section of a wire-bond power semiconductor module.

Drawbacks of these methods are increased system complexity and increased level of required understanding for the same accuracy.

In this work, we propose wireless, low power, single chip temperature sensors with on-chip coils as a solution for performing condition monitoring on power semiconductors. For this type of sensor, the on-chip coil is a critical component. As demonstrated by von Arx and Najafi, it is possible to achieve good power transfer efficiency from an external reader to a carefully designed on-chip coil [6]. An initial attempt at producing a prototype on-chip coil is demonstrated in this work, and experiments performed on it provides some insight in problems that can arise.

The paper is organised as follows. In Section II the concept of wireless monitoring of temperature for power semiconductors is described. Section III describes experiments on a prototype coil with results in Section IV. A discussion of the results are presented in Section V and the paper is concluded in Section VI.

\section{Wireless Temperature Measurement in Power SEMICONDUCTOR MODULES}

Shown in Fig. 1 is an illustration of the cross-section of a wire-bond power semiconductor module, which is the focus of this work. Other types of modules, such as the press pack module [7], are not considered in this work.

The base in a wire-bond power semiconductor module is a ceramic substrate, called the base plate, onto which power semiconductors are soldered. One purpose of the base plate is to cool the power semiconductors. A metal plate in contact with the base plate is usually exposed at the bottom of the module so that it can be mounted on a heat sink. 


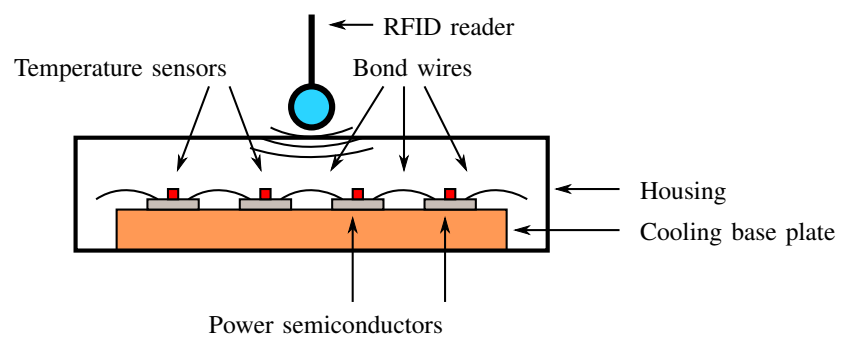

Fig. 2. Schematic view of the cross-section of a wire-bond power semiconductor module whose devices are being monitored by single-chip temperature sensors powered by a nearby RFID reader.

To be able to accurately monitor the temperature of a power semiconductor device while providing galvanic isolation, we propose glueing a single-chip sensor directly onto power semiconductors and communicate to the sensor through a wireless technology such as radio frequency identification (RFID). The concept is illustrated in Fig. 2 and requires the sensor chip to include an on-chip coil. The small size and lack of electrical connections of an integrated circuit with on-chip coil enable multiple sensors to be glued onto a single power semiconductor. This enables measurements of the spatial distribution of temperature over the power semiconductor, which could make faults such as solder fatigue easier to predict as compared to measuring the temperature at a single point on the power device.

There are several options for the placement of the reader coil. In Fig. 2, the reader is mounted on the outside of the module housing. This enables easy integration of the coil with existing modules since no modifications need to be made inside the module except for adding temperature sensors which do not require any extra wires. Another advantage is that the reader coil can be easily aligned the with on-chip coils of the sensors. Such a coil could either be a wire wound coil or a printed circuit board (PCB) coil. Another possibility is to integrate the coil within the power semiconductor module. While this approach requires some modification to existing modules, it has the advantage of closer distance between reader and sensor. Depending on the dimensions of the module, this approach may be necessary to achieve a sufficiently high power transfer efficiency between reader and temperature sensors.

There are several aspects that affect the electromagnetic situation. These include:

1) The substrate of the sensor chip. Currents induced in the substrate reduce the strength of transmitted signals.

2) The power semiconductor. Currents induced in he substrate of the power semiconductor reduce the strength of transmitted signals. In addition to this, the on and off switching of currents typically present in power semiconductors produce strong electromagnetic fields that may disturb the communication between reader and sensor.

3) The base plate. Currents induced in the base plate reduce the strength of transmitted signals.
4) The spatial separation between sensor and reader. A larger distance between reader and sensor reduces the power transfer efficiency. This distance is determined by the dimensions of the power semiconductor module and the positioning of the reader coil.

Since power semiconductors are commonly operated at high temperatures and since power is supplied wirelessly for this type of monitoring, the concept requires high temperature, low power temperature sensors such as the sub- $\mu \mathrm{W}$ sensor demonstrated in [8].

Although the electromagnetic environment in a power semiconductor module is complex, an indication of the feasibility can be seen from works on-chip coils in other environments. For example, in a work on implantable chips in humans, wireless transfer efficiencies in air of better than $1 \%$ at a distance of $10 \mathrm{~mm}$ have been demonstrated [6] and its $2.18 \mathrm{~mm} \times 2.18 \mathrm{~mm}$ on-chip coil could be used in conjunction with the $450 \mathrm{nW}$ sensor from [8] to implement the condition monitoring scheme proposed in this work.

\section{ON-CHIP COIL PROTOYPE}

In contrast to [6] where power levels in the range of 1 to $10 \mathrm{~mW}$ were achieved, we foresee significantly lower power requirements in our applications. As demonstrated in [8], accurate temperature sensors consuming as little as $450 \mathrm{nW}$ are possible to implement. However, to leave some margin for other on-chip circuits such as A/D conversion, communication and control circuits, our first attempt at building an onchip coil targeted a total power budget of $40 \mu \mathrm{A}$ at $2 \mathrm{~V}$, or $80 \mu \mathrm{W}$. The intended operating frequency of this prototype was $13.56 \mathrm{MHz}$, which is often used for RFID applications. As we found it to be impossible to build an on-chip coil with any significant Q-value for this frequency, the coil was instead optimised so that the resistance of the coil approximately matches the load impedance $(2 \mathrm{~V} / 40 \mu \mathrm{A}=50 \mathrm{k} \Omega)$ of the onchip circuits. The result was a $1.525 \mathrm{~mm} \times 1.525 \mathrm{~mm}$ square coil with 199 turns and an inductance of approximately $150 \mu \mathrm{H}$ $\left(X_{L}=12.8 \mathrm{k} \Omega, Q=0.26\right)$. As our intended operating frequency is significantly lower than that used in [6] $(160 \mathrm{MHz})$, significantly lower power transfer efficiency can be expected. The coil was manufactured in $180 \mathrm{~nm}$ CMOS with no special post-processing steps carried out to manufacture the coil. Such post-processing can be used in order to produce higher quality coils by for example electroplating a $\mathrm{Cu}$ winding and a $\mathrm{NiFe}$ core onto a semiconductor chip [9], but this work focuses instead on cheaper coils that do not require such processing.

The remainder of this section describes two experiments that were performed on this prototype chip coil. A schematic of the experiment setup is shown in Fig. 3. $L_{\text {chip }}$ represents the on-chip coil, while $L_{\text {reader }}$ represents the reader coil. Actual power semiconductor chips were not included in this setup.

\section{A. Chip coil parameter estimation}

The left-hand part of the circuit shown in Fig. 3 was used for this experiment. A frequency sweep of the voltage, $v_{i 1}(t)$, was performed and the resulting waveforms, $v_{i 1}(t)$ and $v_{o 1}(t)$, 


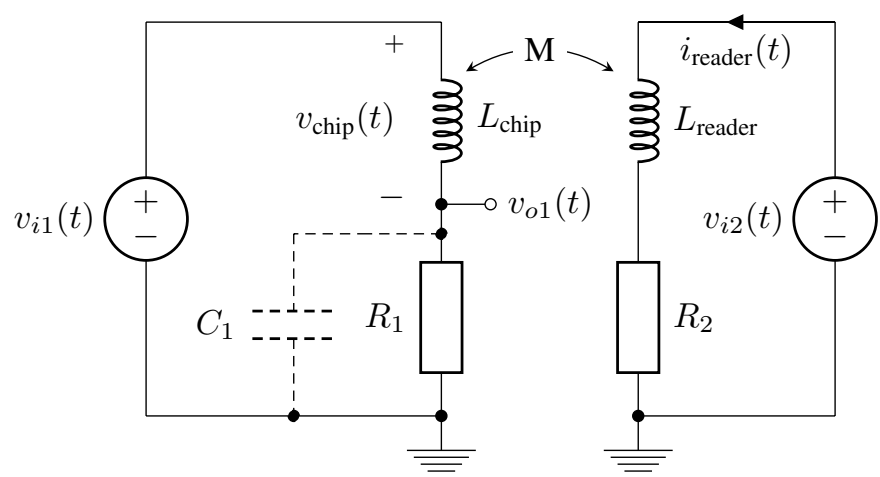

Fig. 3. Schematic of the experiment setup. The left-hand side represents the chip coil, $L_{\text {chip }}$ connected in series with the resistor $R_{1}$ and the signal generator $v_{i 1}(t)$. The right-hand side represents the reader coil, $L_{\text {reader }}$, coupled to the chip coil, $L_{\text {chip }}$, with mutual inductance, $M$ and connected in series with the resistor $R_{2}$ and the signal generator, $v_{i 2}(t) . C_{1}$ represents the stray capacitance caused by the circuit board and the measurment equipment.

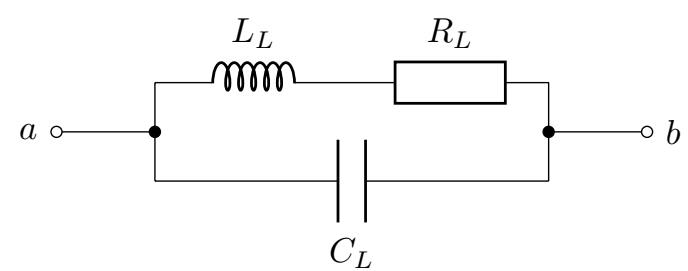

Fig. 4. Equivalent model for a coil inductor.

were sampled. A Fourier transform was taken on the acquired samples to obtain the phasors $\mathbf{V}_{\mathbf{i} \mathbf{1}}(j \omega)$ and $\mathbf{V}_{\mathbf{o} \mathbf{1}}(j \omega)$. From the schematic shown in Fig. 3 with the model of the coil inductor shown in Fig. 4 replacing $L_{\text {chip }}$ and taking into account stray capacitance, $C_{1}$, in parallel with $R_{1}$, the voltage division from $\mathbf{V}_{\mathbf{i} \mathbf{1}}(j \omega)$ to $\mathbf{V}_{\mathbf{o 1}}(j \omega)$ yields

$$
\frac{\mathbf{V}_{\mathbf{o} 1}(j \omega)}{\mathbf{V}_{\mathbf{i} 1}(j \omega)}=\frac{Z_{1}}{Z_{1}+Z_{\text {chip }}},
$$

where

$$
Z_{1}=R_{1} \| \frac{1}{j \omega C_{1}}
$$

and

$$
Z_{\text {chip }}=\left(R_{L_{\text {chip }}}+j \omega L_{L_{\text {chip }}}\right) \| \frac{1}{j \omega C_{L_{\text {chip }}}} .
$$

Taking the real and imaginary parts on both sides of Equation 1 produces two new equations which were used with the sampled data in a Gauss-Newton optimiser to obtain estimates of the coil parameters $R_{L_{\text {chip }}}, L_{L_{\text {chip }}}$ and $C_{L_{\text {chip }}}$.

\section{B. Power transfer efficiency}

To assess the power transfer efficiency from an external coil to the chip coil, a reader coil was manufactured according to the specifications given in Table I. The separation between reader coil and chip coil was set to $7 \mathrm{~mm}$. Consider the circuit shown in Fig. 3. The voltage source $v_{i 1}(t)$ and the resistor $R_{1}$ were replaced by open circuits and the induced voltage, $v_{\text {chip }}(t)$, over $L_{\text {chip }}$ was measured for different frequencies on
TABLE I

SPECIFICATIONS FOR THE READER COIL USED IN THIS WORK.

\begin{tabular}{lc} 
Paremeter & Value \\
\hline \hline Wire material & Copper \\
Wire diameter & $0.3 \mathrm{~mm}$ \\
Coil diameter & $10 \mathrm{~mm}$ \\
Coil height & $8 \mathrm{~mm}$ \\
Number of turns & 25
\end{tabular}

the voltage source $v_{i 2}(t)$. The current, $i_{\text {reader }}(t)$, through the reader coil was also monitored by measuring the voltage over resistor $R_{2}$

If the reader circuit is implemented as a resonant circuit driven by a power amplifier, the dominant resistance is that of the reader coil, $R_{L_{\text {reader }}}$, and the losses in the reader will be given by:

$$
P_{\text {loss }}=I_{\text {reader, rms }}^{2} R_{L_{\text {reader }}},
$$

where $I_{\text {reader, rms }}$ is the root-mean-square (rms) current passing through the reader coil.

The maximum achievable power transferred to a circuit connected to the chip coil occurs when the resistance of that circuit is matched to that of the resistance of chip coil, $R_{L_{\text {chip }}}$. This power is given by:

$$
P_{\text {useful }}=\frac{V_{\text {chip, rms }}^{2}}{4 R_{L_{\text {chip }}}},
$$

where $V_{\text {chip, rms }}$ is the rms value of the voltage induced in the reader coil, $L_{\text {reader }}$.

Combining Equation 2 and Equation 3 yields

$$
\eta_{\text {max }}=\frac{P_{\text {useful }}}{P_{\text {loss }}}=\frac{1}{4 R_{L_{\text {reader }}} R_{L_{\text {chip }}}} \frac{V_{\text {chip, rms }}^{2}}{I_{\text {reader, rms }}^{2}},
$$

where $\eta_{\max }$ is the power transfer efficiency with matched load resistance.

\section{RESULTS}

Based on the data obtained from the experiment presented in Section III-A with two frequency sweeps from $10 \mathrm{kHz}$ to $1 \mathrm{MHz}$ for both $R_{1}=1 \mathrm{k} \Omega$ and $R_{1}=47 \mathrm{k} \Omega$, estimations of the inductor parameters, $R_{L_{\text {chip }}}$ and $C_{L_{\text {chip }}}$, were found to be $47.6 \mathrm{k} \Omega$ and $3.42 \mathrm{pF}$, respectively. Gauss-Newton optimisation did not show stable values for the inductance, $L_{L_{\text {chip }}}$, and a closer examination of the data revealed that the circuit showed no significant inductive behaviour. The reason for this is the high values of $R_{L_{\text {chip }}}$ and $C_{L_{\text {chip }}}$. At high frequencies where the inductive part of the impedance starts to become significant compared to the resistance $R_{L_{\text {chip }}}$ the capacitive part becomes dominant and makes the coil act as a short circuit, hindering any efforts to measure the inductance. Electromagnetic simulations support this argumentation by showing a simulated inductance of $150 \mu \mathrm{H}$ which is small enough compared to the obtained capacitance and resistance that the whole circuit does not show any significant inductive behaviour. 
TABLE II

MEASURED AND CALCULATED DATA FOR THE POWER TRANSFER EXPERIMENT EFFICIENCY DESCRIBED IN SECTION III-B. $I_{\text {READER, RMS AND }}$

$V_{\text {CHIP, RMS }}$ ARE THE OBSERVED AMPLIDUDES OF THE READER COIL

CURRENT AND THE CHIP COIL VOLTAGE, RESPECTIVELY. $\eta_{\text {MAX }}$ IS THE CALCULATED POWER TRANSFER EFFICIENCY BASED ON THE

MEASUREMENTS AND ESTIMATIONS FOR THE COIL RESISTANCES.

\begin{tabular}{|c|c|c|}
\hline \multicolumn{3}{|l|}{ Paremeter } \\
\hline Frequency & $125 \mathrm{kHz}$ & $1 \mathrm{MHz}$ \\
\hline \multicolumn{3}{|c|}{ Measured parameters } \\
\hline$I_{\text {reader, rms }}$ & $62.9 \mathrm{~mA}$ & $16.7 \mathrm{~mA}$ \\
\hline$V_{\text {chip, rms }}$ & $3.85 \mathrm{mV}$ & $6.20 \mathrm{mV}$ \\
\hline \multicolumn{3}{|c|}{ Calculated parameters } \\
\hline$\eta_{\max }$ & $0.222 \mathrm{ppm}$ & $8.22 \mathrm{ppm}$ \\
\hline
\end{tabular}

Data for two frequencies were obtained from the experiment presented in Section III-B with a reader-chip separation of $7 \mathrm{~mm}$. This data is summarised in Table II. Measurements at higher frequencies were complicated by the capacitive coupling of the reader coil to the measurement PCB and the obtained data for those frequencies was therefore excluded. It is possible that the effect of capacitive coupling is present in the measurement results also for the lower frequencies and therefore a more precise experiment setup would be beneficial. Table II includes calculated values, using Equation 4, for the power transfer efficiency, $\eta_{\max }$, based on the measured voltages and currents, $I_{\text {reader, rms }}$ and $V_{\text {chip, rms }}$ along with estimations of $R_{L_{\text {chip }}}$ and $R_{L_{\text {reader }}} . R_{L_{\text {chip }}}$ was found to be $45.1 \mathrm{k} \Omega$ through the chip coil parameter estimation process described in earlier in this section, while $R_{L_{\text {reader }}}$ was calculated to be $187 \mathrm{~m} \Omega$ from the specifications of the reader coil, shown in Table I.

\section{Discussion}

Based on a simulated coil inductance of $150 \mu \mathrm{H}$, a total coil impedance $47.6 \mathrm{k} \Omega+j 12.8 \mathrm{k} \Omega$ results. Hence, the large self capacitance of $3.42 \mathrm{pF}$ is clearly much too large for operation at $13.56 \mathrm{MHz}$ as it corresponds to a reactance of about $3.4 \mathrm{k} \Omega$. Thus, a large fraction of the induced voltage would be lost due to the voltage division between $R_{L_{\text {chip }}}+L_{L_{\text {chip }}}$ and $C_{L_{\text {chip }}}$. Considering the behaviour of the power transfer efficiency, $\eta_{\max }$, Table II shows that when increasing the frequency from $125 \mathrm{kHz}$ to $1 \mathrm{MHz}, \eta_{\max }$ increases by a factor 37.0. This differs from the theoretical value for ideal coils of $8^{2}=64$ (since the frequency was increased by a factor of 8 ), again possibly due to voltage division when driving the large self-capacitance. Taking this into account, the theoretical improvement from $125 \mathrm{kHz}$ to $1 \mathrm{MHz}$ was calculated to be a factor 46 , in closer agreement with the measured value.

Optimising the coil to match the load impedance without considering the self-capacitance of the coil resulted in a suboptimal design. Further work will be required to find a design that properly balances both inductance, and thus mutual inductance to the reader coil, with coil resistance and capacitance. Although the present work focused on $13.56 \mathrm{MHz}$, going to higher operating frequencies presently appear advantageous. For example, of the industrial, scientific and medical (ISM) bands currently available in the European Union (EU), the $27 \mathrm{MHz}, 40.7 \mathrm{MHz}$, or $169.4 \mathrm{MHz}$ appear especially suitable due to the high permissible power levels. These bands require duty cycle limitations of $0.1 \%$ to $1 \%$, but this does not need to be a limitation in condition monitoring applications where measurement durations in the millisecond range and repetition rates on time-scale on the order of seconds can be sufficient.

\section{CONCLUSION}

We propose a concept of using wireless, direct-contact sensors with on-chip coils to accurately monitor the temperature of power semiconductors while providing galvanic isolation from them. A prototype chip with on-chip coil was manufactured as an initial step to assess the feasibility of this concept. Said chip demonstrate that attention need to be paid so that the coil capacitance, $C_{L_{\text {chip }}}$, does not become too large for the coil to be effective at high frequencies.

Earlier work that support the feasibility of this concept include [6] which have demonstrated power transfer efficiencies of around $1 \%$ for on-chip coils. The coil of that work could be used with the sub- $\mu \mathrm{W}$ sensor demonstrated in [8] to realise the proposed monitoring scheme.

\section{ACKNOWLEDGEMENT}

The work presented in this paper was funded by Svenska Kraftnät.

\section{REFERENCES}

[1] E. Wolfgang, "Examples for failures in power electronics systems," $E C P E$ tutorial on reliability of power electronic systems, Nuremberg, Germany, 2007.

[2] S. Yang, D. Xiang, A. Bryant, P. Mawby, L. Ran, and P. Tavner, "Condition monitoring for device reliability in power electronic converters: A review," Power Electronics, IEEE Transactions on, vol. 25, no. 11, pp. 2734-2752, Nov 2010.

[3] H. Oh, B. Han, P. McCluskey, C. Han, and B. Youn, "Physics-of-failure, condition monitoring, and prognostics of insulated gate bipolar transistor modules: A review," Power Electronics, IEEE Transactions on, vol. 30, no. 5, pp. 2413-2426, May 2015.

[4] M. Held, P. Jacob, G. Nicoletti, P. Scacco, and M.-H. Poech, "Fast power cycling test of IGBT modules in traction application," in Power Electronics and Drive Systems, 1997. Proceedings., 1997 International Conference on, vol. 1, May 1997, pp. 425-430 vol.1.

[5] D. Barlini, M. Ciappa, M. Mermet-Guyennet, and W. Fichtner, "Measurement of the transient junction temperature in MOSFET devices under operating conditions," Microelectronics Reliability, vol. 47, no. 9-11, pp. 1707 - 1712, 2007, 18th European Symposium on Reliability of Electron Devices, Failure Physics and Analysis.

[6] M. Zargham and P. Gulak, "Fully integrated on-chip coil in $0.13 \mu \mathrm{m}$ cmos for wireless power transfer through biological media," Biomedical Circuits and Systems, IEEE Transactions on, vol. 9, no. 2, pp. 259-271, April 2015

[7] T. Stockmeier, "From packaging to "un"-packaging - trends in power semiconductor modules," in Power Semiconductor Devices and IC's, 2008. ISPSD '08. 20th International Symposium on, May 2008, pp. 12 19.

[8] J. Nilsson, J. Borg, and J. Johansson, "Leakage current compensation for a $450 \mathrm{nW}$, high-temperature, bandgap temperature sensor," in Mixed Design of Integrated Circuits Systems (MIXDES), 2015 22nd International Conference, June 2015, pp. 343-347.

[9] J. von Arx and K. Najafi, "On-chip coils with integrated cores for remote inductive powering of integrated microsystems," in Solid State Sensors and Actuators, 1997. TRANSDUCERS '97 Chicago., 1997 International Conference on, vol. 2, Jun 1997, pp. 999-1002 vol.2. 